OPEN ACCESS

Edited by:

Lourdes Morales-Oyervides,

Universidad Autónoma de

Coahuila, Mexico

Reviewed by:

Samuel Bernardo,

Autonomous University of

Chihuahua, Mexico

Mireille Fouillaud,

Université de la Réunion, France

*Correspondence:

Fernanda Cortez Lopes fernandacortezlopes@gmail.com; fernanda.cortez@ufrgs.br

Specialty section: This article was submitted to

Sustainable Food Processing, a section of the journal

Frontiers in Sustainable Food Systems

Received: 30 July 2020 Accepted: 17 February 2021 Published: 18 March 2021

Citation:

Lopes FC and Ligabue-Braun $R$ (2021) Agro-Industrial Residues:

Eco-Friendly and Inexpensive Substrates for Microbial Pigments

Production

Front. Sustain. Food Syst. 5:589414. doi: 10.3389/fsufs. 2021.589414

\section{Agro-Industrial Residues: Eco-Friendly and Inexpensive Substrates for Microbial Pigments Production}

\author{
Fernanda Cortez Lopes ${ }^{1 *}$ and Rodrigo Ligabue-Braun ${ }^{2}$ \\ ${ }^{1}$ Graduate Program in Cell and Molecular Biology, Center for Biotechnology, Universidade Federal do Rio Grande do Sul, \\ Porto Alegre, Brazil, ${ }^{2}$ Department of Pharmaceutical Sciences, Universidade Federal de Ciências da Saúde de Porto Alegre, \\ Porto Alegre, Brazil
}

Many commodities are abundantly produced around the world, including soybean, corn, rice sugarcane, cassava, coffee, fruits, and many others. These productions are responsible for the generation of enormous amounts of daily residues, such as cassava and sugarcane bagasses, rice husk, and coffee peel. These residues are rich sources for renewable energy and can be used as substrates for industrial interest products. Microorganisms are useful biofactories, capable of producing important primary and secondary metabolites, including alcohol, enzymes, antibiotics, pigments, and many other molecules. The production of pigments was reported in bacteria, filamentous fungi, yeasts, and algae. These natural microbial pigments are very promising because synthetic colorants present a long history of allergies and toxicity. In addition, many natural pigments present other biological activities, such as antioxidant and antimicrobial activities, that are interesting for industrial applications. The use of inexpensive substrates for the production of these metabolites is very attractive, considering that agro-industrial residues are generated in high amounts and usually are a problem to the industry. Therefore, in this article we review the production of microbial pigments using agro-industrial residues during the current decade (2010-2020), considering both submerged and solid state fermentations, wild-type and genetically modified microorganisms, laboratorial to large-scale bioprocesses, and other possible biological activities related to these pigments.

Keywords: microbial pigments, biopigments, agro-industrial residues, biological activities, antimicrobial activity, antioxidant activity

\section{INTRODUCTION}

Color has major importance in human activities. From traffic signals to arts and clothing, color has multiple cultural meanings. It is also central in interpreting edible items regarding their ingestion suitability, that is, if some food or beverage is fresh, ripe, safe, nutritional, or rotten (for instance, red vs. gray beef, green vs. yellow banana) (Sen et al., 2019). 
In this sense, molecules capable of bestowing color to products (food products, in particular) are especially interesting from a technological standpoint. Such molecules are generally called pigments and comprise a variety of chemical structures able to absorb light in the visible range ( $400-700 \mathrm{~nm}$ wavelengths). By chemical definitions, soluble colored substances are called colorants, while insoluble colored substances are called pigments. Nevertheless, under a biological perspective, the colored substances are called pigments, irrespective of their solubility (De Carvalho et al., 2014).

Synthetic colorants have been facing market resistance starting from 1960s due to multiple reasons, including allergenicity, toxicity, teratogenicity, and carcinogenicity problems (Sen et al., 2019). In addition to that, multiple synthetic colorants depend on precursors that are petroleum-based, a non-renewable resource (Kumar et al., 2015). These restrictions, combined with an increased advertising desire to label products (majorly food) as natural, ecological, biological, or eco-friendly have sparked the study of biopigments (Babitha, 2009). Biological pigments are considered safe if they are non-toxic, non-allergenic, non-carcinogenic, and biodegradable (Sen et al., 2019).

Among biological pigment sources, microbes like bacteria, yeasts, molds, and algae are being targeted as ideal resources to be tapped. Microbial pigments are forefront in colorant development due to their independence from weather conditions, considerable assortment of shades, fast growth, and substrate-dependent cost effectiveness, characteristics considered to be superior in comparison to plant and animalsourced pigments (Lopes et al., 2013; Panesar et al., 2015). Such advantages, combined with shifts in consumer preferences, have been responsible for a steep rise in the biopigment market, with natural colors expected to grow $7 \%$ annually as a category (De Carvalho et al., 2014; Sen et al., 2019).

Microbial pigments are especially interesting when one considers that the major cost regarding their production is related to microbes growth media. Thus, as a rule of thumb, low-cost medium translates into low-cost pigment (Panesar et al., 2015; Sen et al., 2019). Among such low-cost media, agro-industrial residues are being regarded as ideal substrates for microbial pigment production, serving as sources of carbon, nitrogen, and minerals (Lopes et al., 2013; Panesar et al., 2015; Sen et al., 2019).

The agro-industrial residues are defined as many different wastes from the food and agriculture industry. These residues include multiple plant-based materials, such as straws, stems, stalks, leaves, husks, shells, peels, lint, seeds, pulps, stubbles, bagasse, spent coffee grounds, brewer's spent grains, and some animal byproducts, including feathers and whey (Madeira et al., 2017; Venil et al., 2020a). The efficient transformation of these residues has become a central environmental issue in recent years, with great attention being given to energy generation (e.g., biodiesel production) (Vandamme, 2009). However, new materials, chemicals and valuable products in general are being obtained from agro-industrial waste, including pigments (Madeira et al., 2017). These added-value products are obtained from cheaper materials that otherwise would cause environmental damage, either directly (phenolic or via other toxic compounds) or indirectly (by changing nutritional aspects of the ecosystem, normally causing eutrophication).

Microbial pigments are already used for food and textile coloring, with proposed uses in candles, soaps, ballpoint, salmon, yogurt, and highlighter pens (Venil et al., 2013; Sen et al., 2019). The applications of microbial-derived pigments, however, are not restricted to coloring. Some of these compounds have additional properties, such as antioxidant, antiparasitic, antimicrobial, and anticancer (Venil et al., 2013, 2020a; Sen et al., 2019).

In this article, we review the current literature on microbial pigment production using agro-industrial residues as substrates, encompassing the last decade (2010-2020). Exceptions were made for cornerstone articles and chapters that were considered of major interest for the reader but were outside the selected timeframe.

\section{PIGMENT-PRODUCING MICROORGANISMS}

Microorganisms used in the production of biopigments include bacteria, yeasts, molds, and algae. The term "microbial pigments" is somewhat vague and can include some species that would not be considered de facto microorganisms (such as some filamentous fungi). In general, microbial pigment defines any non-plant, non-animal sourced biological pigment.

The microorganisms targeted for pigment production must satisfy a series of criteria: they should be non-pathogenic, nontoxic, able to use a wide range of carbon and nitrogen sources, able to give reasonable color yield, be tolerant to high salt concentration, be tolerant to variable temperatures and $\mathrm{pH}$. They must also produce pigments that are easy to extract (Babitha, 2009; Kumar et al., 2015; Panesar et al., 2015; Venil et al., 2020a).

Biopigments can be classified based on chemical structure, with the main representatives being canthaxanthin, astaxanthin, prodigiosin, phycocyanin, violacein, riboflavin, $\beta$-carotene, melanin, and lycopene (Malik et al., 2012; Sen et al., 2019). All of them are currently used in the food industry (Sen et al., 2019). A variety of microbial pigments for which molecular identities were determined are shown in Table 1, along with synthesizing organism and pigment color. The molecular structure for some of the listed pigments is shown in Figure 1. For an updated list of macroscopic fungal pigments, please refer to Lagashetti et al. (2019).

\section{AGRO-INDUSTRIAL RESIDUES USED FOR THE PRODUCTION OF MICROBIAL PIGMENTS}

There is a need to explore novel strains of microorganisms and appropriate strategies for commercial production of microbial pigments (Nigam and Luke, 2016). Since synthetic culture media are usually expensive, the use of agro-industrial waste would be a profitable mean of reducing the production cost (Panesar et al., 2015). The Food and Agriculture Organization (FAO), in 2013, reported that 250 million tons of agro-industrial wastes are generated globally per year, during processing of different plant 
TABLE 1 | Microbial pigments and their source organisms [based on data from Malik et al. (2012), Panesar et al. (2015), Lagashetti et al. (2019), and Sen et al. (2019)].

\begin{tabular}{|c|c|c|}
\hline Pigment (class) & Color & Microorganisms \\
\hline Ankaflavin & Orange & Monascus purpureus \\
\hline Anthraquinone & Red & Pacilomyces farinosus, Penicillium oxalicum, \\
\hline Arpink Red & Dark red & Penicillium oxalicum \\
\hline Astaxanthin & Pink red & $\begin{array}{l}\text { Agrobacterium aurantiacum, Haematococcus pluvialis, Paracoccus carotinifaciens, Xanthophyllomyces dendrorhous } \\
\text { (formerly Phaffia rhodozyma) }\end{array}$ \\
\hline Atrovenetin & Yellow & Penicillium melinii \\
\hline Azaphilones & Red & Penicillium purpurogenum, Talaromyces atroroseus \\
\hline Canthaxanthin & Red & Bradyrhizobium sp., Brevibacterium sp., Dietzia maris, Haloferax alexandrinus, Lactobacillus pluvialis, Monascus roseus \\
\hline Carotenoids & Red & $\begin{array}{l}\text { Blakeslea trispora, Dunaliella salina, Fusarium sporotrichioides, Mucor circinelloides, Neurospora crassa, Phycomyces } \\
\text { blakesleeanus, Rhodotorula rubra }\end{array}$ \\
\hline Cycloprodigiosin & Red & Pseudoalteromonas denitrificans \\
\hline Granadaene & Orange red & Streptococcus agalactiae \\
\hline Indigoidine & Blue & Corynebacterium insidiosum \\
\hline Lutein & Yellow & Chlorella spp. \\
\hline Lycopene & Red & Blakslea trispora, Fusarium sporotrichioides \\
\hline Melanin & Dark brown, Black & $\begin{array}{l}\text { Bacillus thuringiensis } \mathrm{H}-14, \text { Cryptococcus sp., Saccharomyces } \\
\text { neoformans var. nigricans, Streptomyces virginiae, } \\
\text { Yarrowia lipolytica }\end{array}$ \\
\hline Monascin & Yellow & Monascus sp. \\
\hline Monascorubramine & Red & Monascus sp. \\
\hline Naphthoquinone & Brownish yellow & Fusarium sp. \\
\hline Naphtoquinone & Dark red & Cordyceps unilateralis \\
\hline Phycocyanin & Blue & Arthrospira sp. (formerly Spirulina sp.), Pseudomonas spp., \\
\hline Phycoerythrin & Red & Porphyridium cruentum \\
\hline Physcion & Yellow & Aspergillus ruber \\
\hline Prodigiosin & Red & $\begin{array}{l}\text { Alteromonas rubra, Pseudoalteromonas rubra, Rugamonas rubra, Serratia sp., Streptomyces sp., Streptoverticillium } \\
\text { rubrireticuli, Vibrio gaogenes }\end{array}$ \\
\hline Prodoginine & Red & Streptoverticillium rubrireticuli \\
\hline Pyocyanin & Blue green & Pseudomonas aeruginosa \\
\hline Riboflavin & Yellow & Ashbya gossypi, Bacillus subtilis \\
\hline Rubrolone & Red & Streptomyces echinoruber \\
\hline Rubropunctatin & Orange & Monascus sp. \\
\hline Staphyloxanthin & Golden yellow & Staphylococcus aureus \\
\hline Torularhodin & Orange red & Rhodotorula glutinis \\
\hline Violacein & Violet & Chromobacter violaceum, Janthinobacterium lividum, Pseudoalteromonas spp. \\
\hline Xanthomonadin & Yellow & Xanthomonas oryzae \\
\hline Zeaxanthin & Yellow & Flavobacterium sp., Paracoccus zeaxanthinifaciens, Sphingobacterium multivorum, Staphylococcus aureus \\
\hline
\end{tabular}

crops, mainly cereals, starchy roots, fruits, and other vegetables (Heredia-Guerrero et al., 2017).

Natural raw materials and by-products generated by industry are widely used as culture medium due to their low cost, once the components of the medium can represent from 38 to $73 \%$ of the total production cost (Panesar et al., 2015). Agro-industrial residues are untreated and underutilized, while being rich in nutrient components, such as carbohydrates, proteins, fibers, minerals, and vitamins. The utilization of this waste not only eliminates the disposal problems, but also the environment pollution (toxicity to aquatic life, pollution of surface and ground waters, altered soil quality, phyto-toxicity, odorous, and colored natural waters) and negative impact on human and animal health (Panesar et al., 2015; Zihare et al., 2018; Nayak and Bhushan, 2019). The environmental concern is related to the content of these wastes, since most of them include phenolic compounds that have toxic potential, in addition these wastes exhibit high value of biological oxygen demand, chemical oxygen demand, and other suspended solids that can be considered pollutant to the environment (Sadh et al., 2018; Venil et al., 2020a). Furthermore, plant cell walls found in the agro-industrial residues are composed of lignocellulose, a recalcitrant component, consisting of cellulose, hemicellulose and lignin, that can also be an environment pollutant (Sánchez, 2009). Recovery of high value-added components from the waste and their re-utilization as food additives or therapeutics are another interesting aspect to valorize these agro-industrial residues, we will not focus on 
<smiles>CC=CC1=CC2=CC3=C(C(=O)CCCCC)C(=O)OC3(C)C(=O)C2=CO1</smiles><smiles>Cn1c2cccc(=O)c-2nc2ccccc21</smiles>

Pyocyanin<smiles>N=C1C=C(c2cc(N)c(=O)[nH]c2O)C(=O)NC1=O</smiles>

Indigoidine<smiles>O=C1N=c2ccccc2=C1c1cc(-c2c[nH]c3ccc(O)cc23)[nH]c1O</smiles>

Violacein<smiles>CC1=c2[nH]cc3c(c4c5[nH]cc4c(c23)C=5C)C(=O)C1=O</smiles>

Melanin

FIGURE 1 | Chemical structure of some representative microbial pigments.

this approach in the present review. For more information about this strategy, please refer to Nayak and Bhushan (2019). A representative scheme of the different steps for microbial pigment production using agro-industrial waste is proposed in Figure 2.

In this work we reviewed the literature from 2010 to 2020 and selected studies with the production of microbial pigments using agro-industrial residues (Table 2). We restricted our selection to focus on the microorganisms that can produce pigments in an inexpensive manner using agro-industrial residues that are alternatives to decrease the costs of production. During this decade, there were several works using waste as substrate, the sole substrate or supplemented with nutrients. The microorganisms more commonly used were fungi, both molds and yeasts. It is important to highlight some genera such as Monascus and Rhodotorula that were the most prevalent in these studies, especially Monascus purpureus, Rhodotorula glutinis, and R. mucilaginosa. Furthermore, carotenoids and Monascuspolyketides were thus the pigments commonly produced by these microorganisms.

Yeasts are unicellular and have high growth rates, which favor these microorganisms in the production of biopigments (Bhosale and Gadre, 2001). Yeasts are pigment producers 


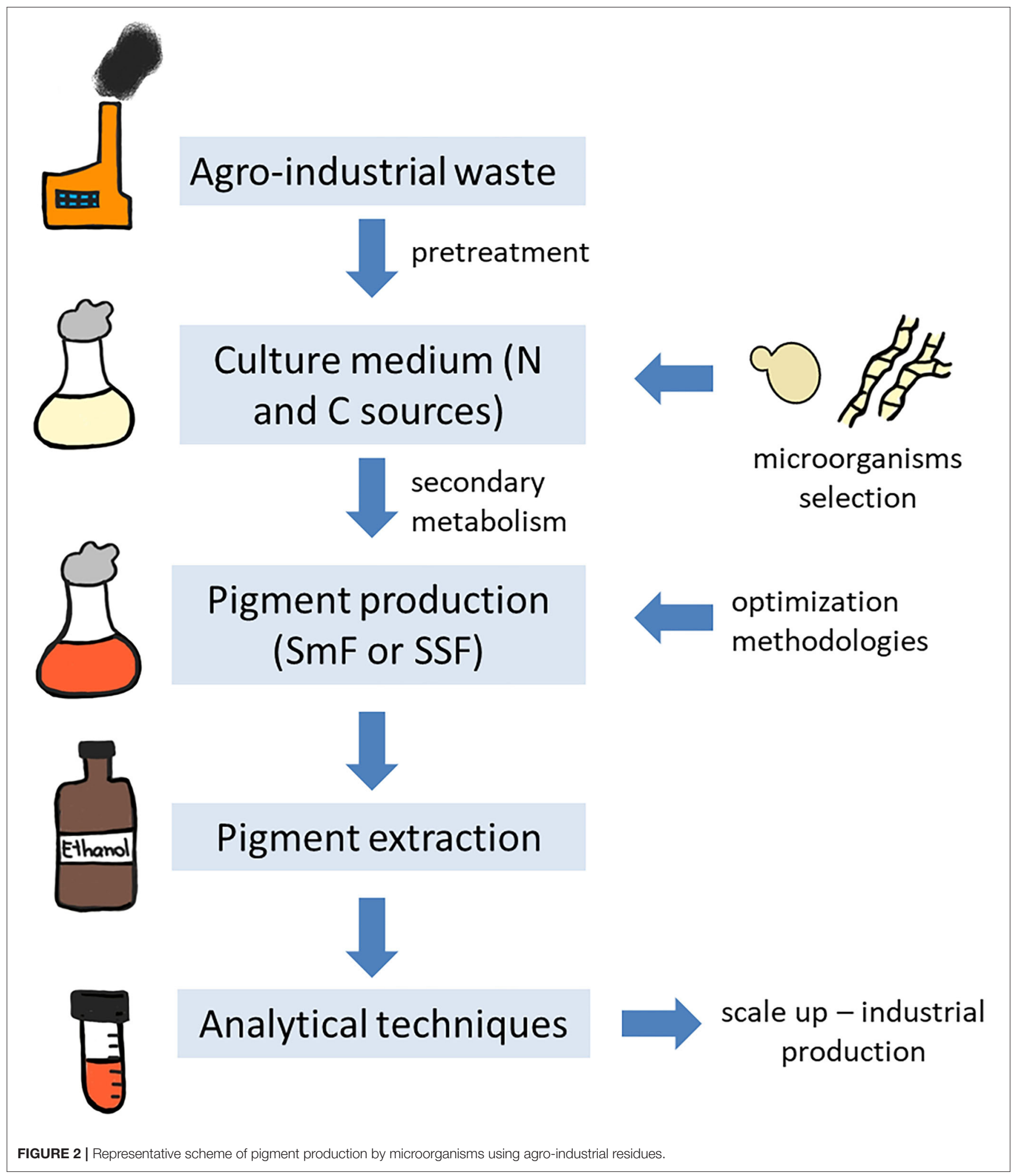

considered as safer in comparison to filamentous fungi, due to the concerns about mycotoxin production by some of the latter. For instance, Monascus spp. is well-known for the production of citrinin, a nephrotoxic and hepatotoxic mycotoxin (Blanc et al., 1995). However, some genetic studies were published, currently modifying genes, eliminating non-essential genes of filamentous 
TABLE 2 | Microbial pigments produced with agro-industrial waste and fermentation conditions (2010-2020).

\begin{tabular}{|c|c|c|c|c|c|c|c|}
\hline $\begin{array}{l}\text { Agro-industrial } \\
\text { residues }\end{array}$ & Microorganisms & Type & Pigment & Fermentation & Scale & Additional information & References \\
\hline Petiole oil palm fronds & $\begin{array}{l}\text { Monascus purpureus } \\
\text { FTC } 5357\end{array}$ & Filamentous fungus & Red pigments & SSF & Laboratory & $\begin{array}{l}\text { Peptone to supplement, } 30^{\circ} \mathrm{C}, 8 \text { days, } 75 \% \\
\text { moisture, yield: } 207 \mathrm{AU} / \mathrm{g} . \mathrm{d}\end{array}$ & Daud et al., 2020 \\
\hline $\begin{array}{l}\text { High test } \\
\text { molasses/sweet } \\
\text { whey/corn steep liquor }\end{array}$ & $\begin{array}{l}\text { Erwinia uredovora } \\
\text { DSMZ } 30080 \\
\text { and Rhodotorula } \\
\text { glutinis } \\
\text { number } 32\end{array}$ & Bacterium and yeast & Carotenoids & SmF & $\begin{array}{l}\text { Laboratory } \\
(100 \mathrm{ml})\end{array}$ & $\begin{array}{l}150 \mathrm{rpm}, 4 \text { days, } 30^{\circ} \mathrm{C} \text {, dark improved } \\
\text { carotenoid production, yield: } 1.46 \pm 0.02 \mathrm{mg} / \mathrm{L}\end{array}$ & Galal and Ahmed, 2020 \\
\hline Peanut seed oil & $\begin{array}{l}\text { Serratia marcescens } \\
11 \mathrm{E}\end{array}$ & Bacterium & Prodigiosin & SmF & $\begin{array}{l}\text { Laboratory } \\
(100 \mathrm{ml})\end{array}$ & $28^{\circ} \mathrm{C}, 150 \mathrm{rpm}, 36 \mathrm{~h}$, yield: $2 \mathrm{~g} / \mathrm{L}$ & $\begin{array}{l}\text { Hernández-Velasco } \\
\text { et al., } 2020\end{array}$ \\
\hline $\begin{array}{l}\text { Paper mill } \\
\text { sludge/sugarcane } \\
\text { bagasse }\end{array}$ & Planococcus sp. TRC1 & Bacterium & $\beta$-carotene & SSF & Laboratory (100 g) & $\begin{array}{l}\text { Supplemented with minimal salt media and } \\
\text { yeast extract, } 30^{\circ} \mathrm{C}, 120 \mathrm{~h} \text {, mixing every } 12 \mathrm{~h} \text {, } \\
80 \% \text { moisture, yields: } 38.54 \pm 1.4 \mathrm{mg} / \mathrm{g} \text { and } \\
47.13 \pm 1.9 \mathrm{mg} / \mathrm{g}\end{array}$ & Majumdar et al., 2020 \\
\hline $\begin{array}{l}\text { Onion peels/mung } \\
\text { bean husk }\end{array}$ & $\begin{array}{l}\text { Rhodotorula mucilaginosa } \\
\text { MTCC-1403 }\end{array}$ & Yeast & $\begin{array}{l}\beta \text {-carotene, } \\
\text { phytoene, } \\
\text { torulene and } \\
\text { Torularhodin }\end{array}$ & SmF & $\begin{array}{l}3 \mathrm{~L} \text { bioreactor } \\
\text { stirred tank }\end{array}$ & $\begin{array}{l}\mathrm{pH} 6.1,25.8^{\circ} \mathrm{C}, 119.6 \mathrm{rpm}, 84 \mathrm{~h}, 1.0 \mathrm{vvm} \text {, } \\
\text { yield: } 719.69 \mu \mathrm{g} / \mathrm{g}\end{array}$ & $\begin{array}{l}\text { Sharma and Ghoshal, } \\
2020\end{array}$ \\
\hline Maltose syrup & $\begin{array}{l}\text { Monascus ruber CCT } \\
3802\end{array}$ & Filamentous fungus & $\begin{array}{l}\text { Orange, yellow } \\
\text { and red } \\
\text { pigments }\end{array}$ & $\begin{array}{l}\text { SmF and } \\
\text { SSF }\end{array}$ & $\begin{array}{l}\text { Laboratory (petri } \\
\text { dishes and } 120 \mathrm{ml} \text { ) }\end{array}$ & $\begin{array}{l}\text { pH influenced the pigment production, } 30^{\circ} \mathrm{C} \text {, } \\
120 \mathrm{rpm}, 192 \mathrm{~h}\end{array}$ & De Oliveira et al., 2019 \\
\hline $\begin{array}{l}\text { Fruits and vegetable } \\
\text { waste }\end{array}$ & $\begin{array}{l}\text { Blakeslea trispora } \\
\text { MTCC884 }\end{array}$ & Filamentous fungus & Carotenoids & SSF & Laboratory & $28^{\circ} \mathrm{C}, 4$ days, $200 \mathrm{rpm}$, yield: $0.127 \mathrm{mg} / \mathrm{mL}$ & Kaur et al., 2019 \\
\hline Bengal gram husk & $\begin{array}{l}\text { Talaromyces } \\
\text { purpureogenus } \\
\text { CFRM02 }\end{array}$ & fungus & Red pigments & SmF & $\begin{array}{l}\text { Laboratory } \\
(100 \mathrm{ml})\end{array}$ & $\begin{array}{l}\mathrm{pH} 5.5,12 \mathrm{~h} \text { day and night, } 30^{\circ} \mathrm{C}, 10 \text { days, } 110 \\
\text { rpm, yield: } 0.565 \pm 0.05 \mathrm{AU} / \mathrm{mL}\end{array}$ & Pandit et al., 2019 \\
\hline Oil palm frond & $\begin{array}{l}\text { Monascus purpureus } \\
\text { FTC } 5356\end{array}$ & fungus & Red pigments & SSF & Laboratory & $\begin{array}{l}50 \% \text { moisture, } \mathrm{pH} 6,2 \% \text { peptone, } 100 \% \\
\text { petiole, and } 10^{8} \mathrm{spores} / \mathrm{mL}, 8 \text { days, } 30^{\circ} \mathrm{C} \text {, } \\
\text { yield: } 2.93 \mathrm{AU} / \mathrm{g}\end{array}$ & Said and Hamid, 2019 \\
\hline Cassava wastewater & $\begin{array}{l}\text { Rhodotorula glutinis } \\
\text { CTT } 2182\end{array}$ & Yeast & Carotenoids & $\mathrm{SmF}$ & $\begin{array}{l}\text { Laboratory } \\
(150 \mathrm{ml})\end{array}$ & $\begin{array}{l}30^{\circ} \mathrm{C}, 200 \mathrm{rpm}, 120 \mathrm{~h} \text {, darkness, yield: } 0.98 \\
\mathrm{mg} / \mathrm{L}\end{array}$ & $\begin{array}{l}\text { Santos Ribeiro et al., } \\
2019\end{array}$ \\
\hline $\begin{array}{l}\text { Mesquite pods/corn } \\
\text { steep liquor }\end{array}$ & $\begin{array}{l}\text { Xanthophyllomyces } \\
\text { dendrorhous ATCC } \\
24202\end{array}$ & Yeast & Carotenoids & SmF & Laboratory (25 ml) & $\begin{array}{l}\text { Mesquite pods extract (2\%), corn steep liquor } \\
(0.3 \%) \text { and yeast extract }(0.3 \%), \mathrm{pH} 5.5,120 \mathrm{~h} \text {, } \\
200 \mathrm{rpm}, 20^{\circ} \mathrm{C} \text {, yield: } 293.41 \pm 31.12 \mu \mathrm{g} / \mathrm{g}\end{array}$ & $\begin{array}{l}\text { Villegas-Méndez et al., } \\
2019\end{array}$ \\
\hline Sugarcane juice & Rhodotorula rubra 102 & Yeast & Carotenoids & $\mathrm{SmF}$ & $\begin{array}{l}\text { Laboratory } \\
(250 \mathrm{ml})\end{array}$ & $\begin{array}{l}1.9 \% \text { reducing sugar, } 2 \% \text { sucrose-and } \\
\text { maltose-based media, } \mathrm{Mg}^{2+}(0.16 \% \text { and } \\
0.196 \%), 30^{\circ} \mathrm{C}, 200 \mathrm{rpm} \text { and } 1,600 \mathrm{Im} \text { of } \\
\text { fluorescent lighting for } 72 \mathrm{~h} \text {, yield: } 30.39 \mathrm{mg} / \mathrm{g}\end{array}$ & Bonadio et al., 2018 \\
\hline Corn cob & $\begin{array}{l}\text { Monascus purpureus } \\
\text { ATCC } 16436\end{array}$ & Filamentous fungus & $\begin{array}{l}\text { Orange and red } \\
\text { pigments }\end{array}$ & SSF & Laboratory & $\begin{array}{l}24 \mathrm{~g} \text { corn cobs, } 2.17 \mathrm{M} \text { glycerol, } \mathrm{pH} 4.5,30^{\circ} \mathrm{C} \text {, } \\
10 \text { days, } 150 \mathrm{rpm}, 12 \times 10^{11} \mathrm{spores} / \mathrm{mL} \text {, yield: } \\
133.77 \mathrm{UA} / \mathrm{mL} \text { and } 108.02 \mathrm{UA} / \mathrm{mL}\end{array}$ & Embaby et al., 2018 \\
\hline $\begin{array}{l}\text { Sugarcane bagasse } \\
\text { hydrolysate }\end{array}$ & $\begin{array}{l}\text { Monascus ruber } \\
\text { Tieghem IOC } 2225\end{array}$ & Filamentous fungus & Red pigment & SmF & Laboratory (50 ml) & $\begin{array}{l}30^{\circ} \mathrm{C}, 150 \mathrm{rpm}, 12 \text { days, supplementation with } \\
\text { glucose and cellobiose, darkness, yield: } \\
18.71 \mathrm{AU}\end{array}$ & Hilares et al., 2018 \\
\hline
\end{tabular}




\begin{tabular}{|c|c|c|c|c|c|c|c|}
\hline $\begin{array}{l}\text { Agro-industrial } \\
\text { residues }\end{array}$ & Microorganisms & Type & Pigment & Fermentation & Scale & Additional information & References \\
\hline Waste orange peels & $\begin{array}{l}\text { Monascus purpureus } \\
\text { ATCC } 16365 \text { and } \\
\text { Penicillium } \\
\text { purpurogenum CBS } \\
113139\end{array}$ & Filamentous fungi & $\begin{array}{l}\text { Yellow, orange } \\
\text { and red } \\
\text { pigments }\end{array}$ & $\begin{array}{l}\text { SSF, } \\
\text { Semi-SSF } \\
\text { and SmF }\end{array}$ & Laboratory & $\begin{array}{l}5 \mathrm{~g} \text { orange peels, } 65 \% \text { moisture, } 25^{\circ} \mathrm{C} \text { and } \\
30^{\circ} \mathrm{C} \text {, yields: } 9 \mathrm{AU} / \mathrm{g}, 0.95 \mathrm{AU} / \mathrm{mL}, 0.58 \mathrm{AU} / \mathrm{mL}\end{array}$ & Kantifedaki et al., 2018 \\
\hline $\begin{array}{l}\text { Coffee husk/pulp } \\
\text { extract }\end{array}$ & $\begin{array}{l}\text { Rhodotorula } \\
\text { mucilaginosa CCMA } \\
0156\end{array}$ & Yeast & Carotenoids & SmF & $\begin{array}{l}\text { Laboratory } \\
(300 \mathrm{ml})\end{array}$ & $\begin{array}{l}8.36 \% \text { coffee husk extract, } 0.636 \% \text { glucose, } \\
0.368 \% \text { peptone and } 0.5 \% \text { tween } 80,10^{7} \\
\text { cell/mL, } 28^{\circ} \mathrm{C}, 160 \mathrm{rpm}, 5 \text { days, dark, yields: } \\
16.36 \pm 0073 \mathrm{mg} / \mathrm{L} \text { and } 21.35 \pm 0067 \mathrm{mg} / \mathrm{L}\end{array}$ & Moreira et al., 2018 \\
\hline $\begin{array}{l}\text { Crude glycerol/Corn } \\
\text { maceration water/rice } \\
\text { parboiling water }\end{array}$ & $\begin{array}{l}\text { Sporidiobolus } \\
\text { salmonicolor CBS } \\
2636\end{array}$ & Yeast & Carotenoids & $\mathrm{SmF}$ & $\begin{array}{l}2 \mathrm{~L} \text { bioreactor } \\
\text { Stirred tank (semi- } \\
\text { continuous process) }\end{array}$ & $\begin{array}{l}8 \% \text { crude glycerol, } 8 \% \\
\text { corn maceration water, and } 2 \% \text { rice parboiling } \\
\text { water, } 25^{\circ} \mathrm{C}, \mathrm{pH} 4.0,180 \mathrm{rpm}, 1.5 \mathrm{vvm}, 50 \% \\
\text { working volume, yields: } 34.8 \mathrm{~g} / \mathrm{L} / \mathrm{h} \text { and } 41.4 \\
\mathrm{~g} / \mathrm{L} / \mathrm{h}\end{array}$ & Colet et al., 2017 \\
\hline Cassava bagasse & $\begin{array}{l}\text { Rhodotorula } \\
\text { mucilaginosa }\end{array}$ & Yeast & Carotenoids & SmF & $\begin{array}{l}\text { Laboratory } \\
(100 \mathrm{ml})\end{array}$ & $\begin{array}{l}2 \% \text { cassava bagasse, } \mathrm{pH} 6.0,25^{\circ} \mathrm{C}, 4 \text { days, } \\
\text { yield: } 12.0-12.5 \mathrm{mg} / \mathrm{L} .\end{array}$ & $\begin{array}{l}\text { Manimala and } \\
\text { Murugesan, } 2017\end{array}$ \\
\hline $\begin{array}{l}\text { Wastes of potato chips } \\
\text { manufacturing }\end{array}$ & $\begin{array}{l}\text { Monascus purpureus } \\
\text { Went NRRL } 1992\end{array}$ & fungus & $\begin{array}{l}\text { Red, orange and } \\
\text { yellow pigments }\end{array}$ & SSF & Laboratory (10 g) & $\begin{array}{l}67 \% \text { moisture, } \mathrm{pH} 6.5,1.5 \mathrm{~mm} \text { particle size } \\
\text { and } 2 \% \text { ammonium sulfate, } 140 \times 10^{3} \\
\text { spores } / 10 \mathrm{~g} \text { dry substrate, } 15 \text { days, } 30^{\circ} \mathrm{C} \text {, } \\
\text { darkness, yields: } 126.5,204.7 \text {, and } 322.9 \mathrm{AU} / \mathrm{g}\end{array}$ & $\begin{array}{l}\text { Abdel-Raheam et al., } \\
2016\end{array}$ \\
\hline Liquid pineapple waste & $\begin{array}{l}\text { Chryseobacterium } \\
\text { artocarpi CECT } 8497\end{array}$ & Bacterium & $\begin{array}{l}\text { Yellowish-orange } \\
\text { pigment }\end{array}$ & SmF & $\begin{array}{l}50 \mathrm{~L} \text { - Bioreactor } \\
\text { stirred tank }\end{array}$ & $\begin{array}{l}\text { 20\% liquid pineapple waste, } 12.5 \% \\
\text { L-tryptophan } \\
1.25 \% \mathrm{KH}_{2} \mathrm{PO}_{4}, 30^{\circ} \mathrm{C}, 200 \mathrm{rpm} \text {, a. r. } 10 \\
\text { L/min, } \mathrm{pH} 7.0 \text {, yield: } 152 \mathrm{mg} / \mathrm{L}\end{array}$ & Aruldass et al., 2016 \\
\hline Olive pomace & $\begin{array}{l}\text { Xanthophyllomyces } \\
\text { dendrorhous } \\
\text { ATCC24202 and } \\
\text { Sporidiobolus } \\
\text { salmonicolor ATCC24259 }\end{array}$ & Yeasts & Astaxanthin & SSF & Laboratory (100 g) & $\begin{array}{l}15^{\circ} \mathrm{C}, \mathrm{pH} 4.5,90 \% \text { moisture, } 10^{6} \mathrm{cells} / \mathrm{mL}, 12 \\
\text { days, yield: } 220.24717 .47 \mathrm{mg} / \mathrm{gdp}\end{array}$ & Eryilmaz et al., 2016 \\
\hline Bakery waste & $\begin{array}{l}\text { Monascus purpureus } \\
\text { ATCC } 16365\end{array}$ & Filamentous fungus & $\begin{array}{l}\text { Orange, yellow } \\
\text { and red } \\
\text { pigments }\end{array}$ & $\begin{array}{l}\text { SSF and } \\
\text { SmF }\end{array}$ & Laboratory & $\begin{array}{l}5 \mathrm{~g} \text { bread waste, moisture } 60 \%, 30^{\circ} \mathrm{C}, 100 \mathrm{~mL} \text {, } \\
250 \mathrm{rpm}(\mathrm{SmF}) \text {, yield: } 24 \mathrm{AU} / \mathrm{g} \text { glucose }\end{array}$ & Haque et al., 2016 \\
\hline $\begin{array}{l}\text { Glycerol and soy } \\
\text { peptone bagasses }\end{array}$ & $\begin{array}{l}\text { Serratia marcescens } \\
\text { Xd-1 }\end{array}$ & Bacterium & Prodigiosin & SSF & Laboratory (2g) & $\begin{array}{l}0.17 \% \text { glycerol bagasse, } 033 \% \text { soy peptone } \\
\text { bagasse, moisture } 83.5 \%, 1 \mathrm{~mm} \text { particles of } \\
\text { bagasse, } 28^{\circ} \mathrm{C}, 48 \mathrm{~h} \text { with mixing every } 12 \mathrm{~h} \text {, } \\
\text { yield: } 40.86 \mathrm{~g} / \mathrm{kg}\end{array}$ & Xia et al., 2016 \\
\hline Liquid pineapple waste & $\begin{array}{l}\text { Chromobacterium } \\
\text { violaceum UTM5 }\end{array}$ & Bacterium & $\begin{array}{l}\text { Violacein and } \\
\text { deoxyviolacein }\end{array}$ & SmF & $\begin{array}{l}50 \mathrm{~L} \text {-Bioreactor } \\
\text { stirred tank }\end{array}$ & $\begin{array}{l}\text { 10\% pineapple waste, } 24 \mathrm{~h}, 30^{\circ} \mathrm{C}, 200 \mathrm{rpm} \text {, } \\
\text { a.r. } 10 \mathrm{~L} / \mathrm{min}, \mathrm{pH} 7.0 \text {, yield: } 16256 \pm 440 \mathrm{mg} / \mathrm{L}\end{array}$ & Aruldass et al., 2015 \\
\hline $\begin{array}{l}\text { Sugarcane bagasse } \\
\text { hydrolysate }\end{array}$ & Dietzia maris NIT-D & Bacterium & $\begin{array}{l}\text { Trans- } \\
\text { canthaxanthin } \\
\text { (carotenoid) }\end{array}$ & SmF & Laboratory (50 ml) & $\begin{array}{l}1.5 \% \text { of total reducing sugars, } 2 \% \text { peptone, } \\
0.5 \% \text { yeast extract, } 0.25 \% \mathrm{NaCl} \text { and } 1 \\
\mathrm{mg} / 100 \mathrm{~mL} \text { of glutamic acid, } \mathrm{pH} 5.5,25^{\circ} \mathrm{C}, 5 \\
\text { days, } 120 \mathrm{rpm}, 2 \% \text { inoculum }\end{array}$ & Goswami et al., 2015 \\
\hline
\end{tabular}




\begin{tabular}{|c|c|c|c|c|c|c|c|}
\hline $\begin{array}{l}\text { Agro-industrial } \\
\text { residues }\end{array}$ & Microorganisms & Type & Pigment & Fermentation & Scale & Additional information & References \\
\hline $\begin{array}{l}\text { Raw glycerol/corn } \\
\text { steep liquor/sugarcane } \\
\text { molasses }\end{array}$ & $\begin{array}{l}\text { Sporodiobolus } \\
\text { pararoseus CCT } 7689\end{array}$ & Yeast & Carotenoids & $\mathrm{SmF}$ & $\begin{array}{l}\text { Laboratory } \\
(250 \mathrm{ml})\end{array}$ & $\begin{array}{l}3 \% \text { raw glycerol and } 5.29 \% \text { corn steep liquor } \\
\text { or } 4 \% \text { sugar cane molasses, } 0.65 \% \text { corn steep } \\
\text { liquor, } 25^{\circ} \mathrm{C}, 180 \mathrm{rpm}, 1 \times 10^{7} \mathrm{cell} / \mathrm{mL} 168 \mathrm{~h} \\
\text { darkness, yield: } 520.94 \mu \mathrm{g} / \mathrm{L}\end{array}$ & $\begin{array}{l}\text { Machado and de } \\
\text { Medeiros Burkert, } 2015\end{array}$ \\
\hline $\begin{array}{l}\text { Carob pulp } \\
\text { syrup/sugarcane } \\
\text { molasses }\end{array}$ & $\begin{array}{l}\text { Rhodosporidium } \\
\text { toruloides NCYC } 921\end{array}$ & Yeast & Carotenoids & SmF & $\begin{array}{l}\text { Laboratory } \\
\text { (200 ml) }\end{array}$ & $\begin{array}{l}7.5 \% \text { sugarcane molasses, } 10 \% \text { carob pulp } \\
\text { syrup, pH } 5.5,30^{\circ} \mathrm{C}, 150 \mathrm{rpm} \text {, yield: } 9.79 \\
\mu \mathrm{g} / \mathrm{L} / \mathrm{h}\end{array}$ & Freitas et al., 2014 \\
\hline $\begin{array}{l}\text { Slaughterhouse } \\
\text { wastewater }\end{array}$ & Phormidium autumnale & Microalgae & Carotenoids & $\mathrm{SmF}$ & $\begin{array}{l}\text { Bubble column } \\
\text { bioreactor ( } 2 \mathrm{~L} \text { ) }\end{array}$ & $\begin{array}{l}26^{\circ} \mathrm{C}, \mathrm{pH} 7.6, \mathrm{C} / \mathrm{N} \text { ratio of } 30,1 \mathrm{vm} \text {, } \\
\text { darkness, residence time of } 168 \mathrm{~h} \text {, yield: } \\
107,902.5 \mathrm{~kg} / \text { year }\end{array}$ & Rodrigues et al., 2014 \\
\hline $\begin{array}{l}\text { Raw glycerol/corn } \\
\text { steep liquor/parboiled } \\
\text { rice water }\end{array}$ & $\begin{array}{l}\text { Sporidiobolus } \\
\text { pararoseus }\end{array}$ & Yeast & Carotenoids & $\mathrm{SmF}$ & $\begin{array}{l}\text { Laboratory } \\
(100 \mathrm{ml})\end{array}$ & $\begin{array}{l}4 \% \text { glycerol, } 4 \% \text { corn steep water, } 2 \% \\
\text { parboiled rice water, } 25^{\circ} \mathrm{C}, \mathrm{pH} 4.0,180 \mathrm{rpm} \text {, } \\
\text { yield: } 843 \mu \mathrm{g} / \mathrm{L}\end{array}$ & Valduga et al., 2014 \\
\hline Raw glycerol & $\begin{array}{l}\text { Monascus ruber CCT } \\
3802\end{array}$ & Filamentous fungus & $\begin{array}{l}\text { Yellow, orange } \\
\text { and red } \\
\text { pigments }\end{array}$ & $\mathrm{SmF}$ & $\begin{array}{l}\text { Bioreactor stirred } \\
\operatorname{tank}(4 \mathrm{~L})\end{array}$ & $\begin{array}{l}1 \% \text { glucose, } 1 \% \text { glycerin, } 0.5 \% \text { glycine, } 0.5 \% \\
\mathrm{~K}_{2} \mathrm{HPO}_{4}, 0.5 \% \mathrm{KH}_{2} \mathrm{PO}_{4} \text { and micronutrients, } \\
30^{\circ} \mathrm{C}, 350 \mathrm{rpm}, 1 \mathrm{vvm}, \mathrm{pH} 6.5 \text {, yield: } 8.28 \cup \mathrm{A}\end{array}$ & Bühler et al., 2013 \\
\hline $\begin{array}{l}\text { Grape waste/cheese } \\
\text { whey/soybean } \\
\text { meal/feather meal/soy } \\
\text { protein/rice husk }\end{array}$ & $\begin{array}{l}\text { Penicillium } \\
\text { chrysogenum IFL1 and } \\
\text { IFL2, Fusarium } \\
\text { graminearum IFL3, } \\
\text { Monascus purpureus } \\
\text { NRRL 1992, P. } \\
\text { vasconiae IFL4 }\end{array}$ & Filamentous fungi & $\begin{array}{l}\text { Yellow, orange } \\
\text { and red } \\
\text { pigments }\end{array}$ & $\mathrm{SmF}$ & Laboratory (50 ml) & $\begin{array}{l}1 \% \text { of each waste, } \mathrm{pH} 6.5,7 \text { days, } 30^{\circ} \mathrm{C}, 125 \\
\mathrm{rpm}, 10^{6} \mathrm{spores} / \mathrm{mL}\end{array}$ & Lopes et al., 2013 \\
\hline Brewery wastewater & $\begin{array}{l}\text { Rhodotorula glutinis } \\
\text { ATCC } 15125\end{array}$ & Yeast & Carotenoids & $\mathrm{SmF}$ & $\begin{array}{l}\text { Laboratory } \\
(500 \mathrm{ml})\end{array}$ & $115 \mathrm{rpm}, 25^{\circ} \mathrm{C}, 168 \mathrm{~h}$, yields: 0.6 and $1.2 \mathrm{mg} / \mathrm{L}$ & Schneider et al., 2013 \\
\hline $\begin{array}{l}\text { corn cob waste stream } \\
\text { cellulose }\end{array}$ & $\begin{array}{l}\text { Penicillium } \\
\text { resticulosum }\end{array}$ & Filamentous fungus & Red pigments & $\mathrm{SmF}$ & Laboratory $(50 \mathrm{ml})$ & $\begin{array}{l}12 \text { days, } 25^{\circ} \mathrm{C}, 60-70 \% \text {, relative humidity, } \\
\text { dark, yield: } 497.03 \pm 55.13 \mathrm{mg} / \mathrm{L}\end{array}$ & Sopandi et al., 2013 \\
\hline $\begin{array}{l}\text { Vegetable cabbage } \\
\text { waste }\end{array}$ & Pseudomonas sp. & Bacterium & Melanin & $\mathrm{SmF}$ & Laboratory (50 ml) & $25^{\circ} \mathrm{C}, 200 \mathrm{rpm}, 48-72 \mathrm{~h}, 2.79 \mathrm{mg} / \mathrm{mL}$ & $\begin{array}{l}\text { Tarangini and Mishra, } \\
2013\end{array}$ \\
\hline Sugarcane bagasse & $\begin{array}{l}\text { Chromobacterium } \\
\text { violaceum }\end{array}$ & Bacterium & Violacein & $\mathrm{SmF}$ & Laboratory (50 ml) & $\begin{array}{l}3 \mathrm{~g} \text { sugarcane bagasse, } 10 \% \mathrm{~L} \text {-tryptophan, } \\
200 \mathrm{rpm}, 30^{\circ} \mathrm{C}, 24 \mathrm{~h} \text {, yield: } 0.82 \mathrm{~g} / \mathrm{L}\end{array}$ & Ahmad et al., 2012 \\
\hline Corn meal & $\begin{array}{l}\text { Monascus purpureus } \\
\text { CMU001 }\end{array}$ & Filamentous fungus & Red pigments & SSF & $\begin{array}{l}6 \times 10 \text {-in. plastic } \\
\text { bags }\end{array}$ & $\begin{array}{l}5 \mathrm{~g} \text { of waste, salt solution, } 8 \% \text { glucose, } 30^{\circ} \mathrm{C} \\
\text { for } 14 \text { days, } 1 \times 10^{6} \text { spores } / \mathrm{mL} \text {, yield: } 129.63 \\
\mathrm{U} / \mathrm{gds}\end{array}$ & $\begin{array}{l}\text { Nimnoi and Lumyong, } \\
2011\end{array}$ \\
\hline Waste chicken feathers & $\begin{array}{l}\text { Rhodotorula glutinis } \\
\text { MT-5 }\end{array}$ & Yeast & Carotenoids & $\mathrm{SmF}$ & $\begin{array}{l}\text { Laboratory } \\
(100 \mathrm{~mL})\end{array}$ & $\begin{array}{l}0.8 \% \text { peptone of chicken feather, } 4 \% \text { glucose, } \\
0.4 \% \text { yeast extract, } \mathrm{pH} 6.0,30^{\circ} \mathrm{C}, 200 \mathrm{rpm} \text {, } \\
\text { yield: } 6.47 \mathrm{mg} / \mathrm{g}\end{array}$ & Taskin et al., 2011 \\
\hline Corn cob powder & $\begin{array}{l}\text { Monascus purpureus } \\
\text { KACC } 42430\end{array}$ & Filamentous fungus & $\begin{array}{l}\text { Yellow and red } \\
\text { pigments }\end{array}$ & SSF & Laboratory (5 g) & $\begin{array}{l}60 \% \text { moisture, } 30^{\circ} \mathrm{C}, 4 \mathrm{~mL} \text { of spores/gram of } \\
\text { dry substrate, } 7 \text { days, yield: } 25.42 \mathrm{OD} \\
\text { Units/gram }\end{array}$ & Velmurugan et al., 2011 \\
\hline Rice bran & Rhodotorula glutinis & Yeast & $\beta$-carotene & SSF & Laboratory (5 g) & $\begin{array}{l}\mathrm{pH} 5,70 \% \text { moisture, } \mathrm{C}: \mathrm{N} \text { ratio } 4 \text {, yield: } 2.12 \\
\mathrm{mg} / \mathrm{kg} \text { rice bran }\end{array}$ & $\begin{array}{l}\text { Roadjanakamolson and } \\
\text { Suntornsuk, } 2010\end{array}$ \\
\hline
\end{tabular}


fungi in order to increase the production of pigment and decrease mycotoxin secretion (Lagashetti et al., 2019). In the case of Monascus, several techniques were performed to decrease the production of citrinin: changes in the nitrogen composition of the medium, the dissolved oxygen or the $\mathrm{pH}$, as well as genetic alterations of the strains (Sen et al., 2019).

In the reviewed studies, some investigated the production of citrinin by Monascus strains. M. purpureus Went NRRL 1992 did not produce citrinin using potato waste in a solidstate fermentation. The mycotoxin detection was performed by thin layer chromatography (Abdel-Raheam et al., 2016). $M$. purpureus NRRL 1992 produced citrinin in potato dextrose agar, with mycotoxin production being evaluated by ESIMS/MS (electrospray ionization tandem mass spectrometry). Nevertheless, the authors did not evaluate its production in the agro-industrial residues (Lopes et al., 2013). M. purpureus ATCC 16365 also produced citrinin in orange processing waste, however the authors did not demonstrate the methodology used in this study to detect citrinin (Kantifedaki et al., 2018). The detection of mycotoxin production by filamentous fungi is very important, considering the industrial production of pigments and it is a fundamental step to guarantee the safety of the final product.

Microorganisms from these studies were wild-type, and many of them were purchased from collection cultures or isolates. This fact is surprising due to the great advances regarding mutation techniques and heterologous expression with the purpose of obtaining strain improvement. Strain development is important because pigments produced by wild type strains are usually too low in quantity and take longer fermentation periods, making the process uneconomical (Sen et al., 2019). Due to the recent application of molecular techniques to improve pigment production, more studies will be probably published in a near future with recombinant strains grown on agroindustrial residues.

Regarding the wastes, pre-treatment is important for promoting breakdown of these residues, mainly formed by cellulose, hemicellulose and lignin. This breakdown will increase the availability of the nutrients from the substrate to the microorganisms. Various pre-treatment methods such as physical, chemical, biological (enzymatic), and combined are available (nee'Nigam et al., 2009). In the selected studies, the agro-industrial wastes were pre-treated (treated prior to use). Some examples of physical pre-treatment deal with paper mill sludge and sugarcane bagasse that are milled and sieved to achieve an uniform size of particles (Majumdar et al., 2020), as well as onion peels and mung bean husk (Sharma and Ghoshal, 2020), petiole oil palm fronds (Daud et al., 2020) and fruits and vegetable wastes (Kaur et al., 2019). Chemical treatments of the waste were performed in some studies. Bengal gram husk was pre-treated with hydrochloride acid, promoting an acid hydrolysis to improve the availability of the substrate (Pandit et al., 2019). Sugarcane bagasse was pre-treated under alkaline condition with sodium hydroxide and afterwards hydrolyzed with a commercial cellulase complex (Hilares et al., 2018). In this case there was the combination of a biological treatment. Bakery waste was also hydrolyzed with enzymes produced by Aspergillus awamori and Aspergillus oryzae before its use (Haque et al., 2016). In the majority of the studies, the agro-industrial wastes are used as carbon, nitrogen, and micronutrient sources by microorganisms. However, in some cases, the residues can be added as an inert support for the fermentation process, such as the bagasse used in the prodigiosin production by Serratia marcescens (Xia et al., 2016).

Brazil is one of the biggest agricultural commodity producers, since it is considered as the world's biggest producer of sugar, coffee, orange juice, and soybeans (Da Silva Vilar et al., 2019). In this review, 11 studies were performed in Brazil, due to the great availability of such residues in this country. Wastes used in these works were maltose syrup, cassava wastewater, sugarcane (juice, molasses, and hydrolyzed bagasse), solid coffee waste, crude glycerol, rice parboiling water, slaughterhouse wastewater, corn steep liquor (or corn maceration water), grape waste, cheese whey, soybean meal, feather meal, soy protein, and rice husk) (Table 2). In order to evaluate each residue as carbon and/or nitrogen source, Table 3 brings an average composition of the agro-industrial residues regarding carbon and nitrogen composition, according either to the works cited in this review or, in some cases to the composition obtained from other works, when this information was not available. It is important to highlight that this composition is variable, depending on the source of the waste; however, an average composition can be useful for planning new projects in this area.

\section{MODES OF FERMENTATION AND SCALE OF PRODUCTION}

Traditional methods of microorganism isolation, culture and products extraction are now substituted by novel biotechnological techniques and strategies, via the advent of genetic engineering and fermentation technologies (Nigam and Luke, 2016). Different types of fermentation are used to produce pigments depending on the chosen strain and the type of pigment that will be extracted. Production of pigments by fermentation has a great number of advantages, including abundance of raw materials, absence of seasonal variation, cheaper production, easier extraction, perfectible yields, and procurement of biodegradable pigments (Venil et al., 2013; Charalampia et al., 2017). In addition, pigments produced by microorganisms through fermentation present higher stability to heat, to light exposure and $\mathrm{pH}$ variations, and are highly soluble in water (when compared to plant pigments) (Nigam and Luke, 2016). Some types of pigments are only produced by microorganisms (Dufossé, 2006) and the possibily of using industrially important species, such as Escherichia coli and Saccharomyces cerevisiae, to express these pigments heterologously are excellent alternatives (Venil et al., 2013).

Microorganisms produce pigments mainly by two types of fermentations processes: solid state fermentation (SSF) and submerged fermentation (SmF). In the SSF technique, the substrates are used by the microorganisms very slowly, and then the same substrate can be used for longer periods. This technique provides controlled release of nutrients during the process (Subramaniyam and Vimala, 2012). This type of 
TABLE 3 | Average carbon and nitrogen composition of the agro-industrial residues.

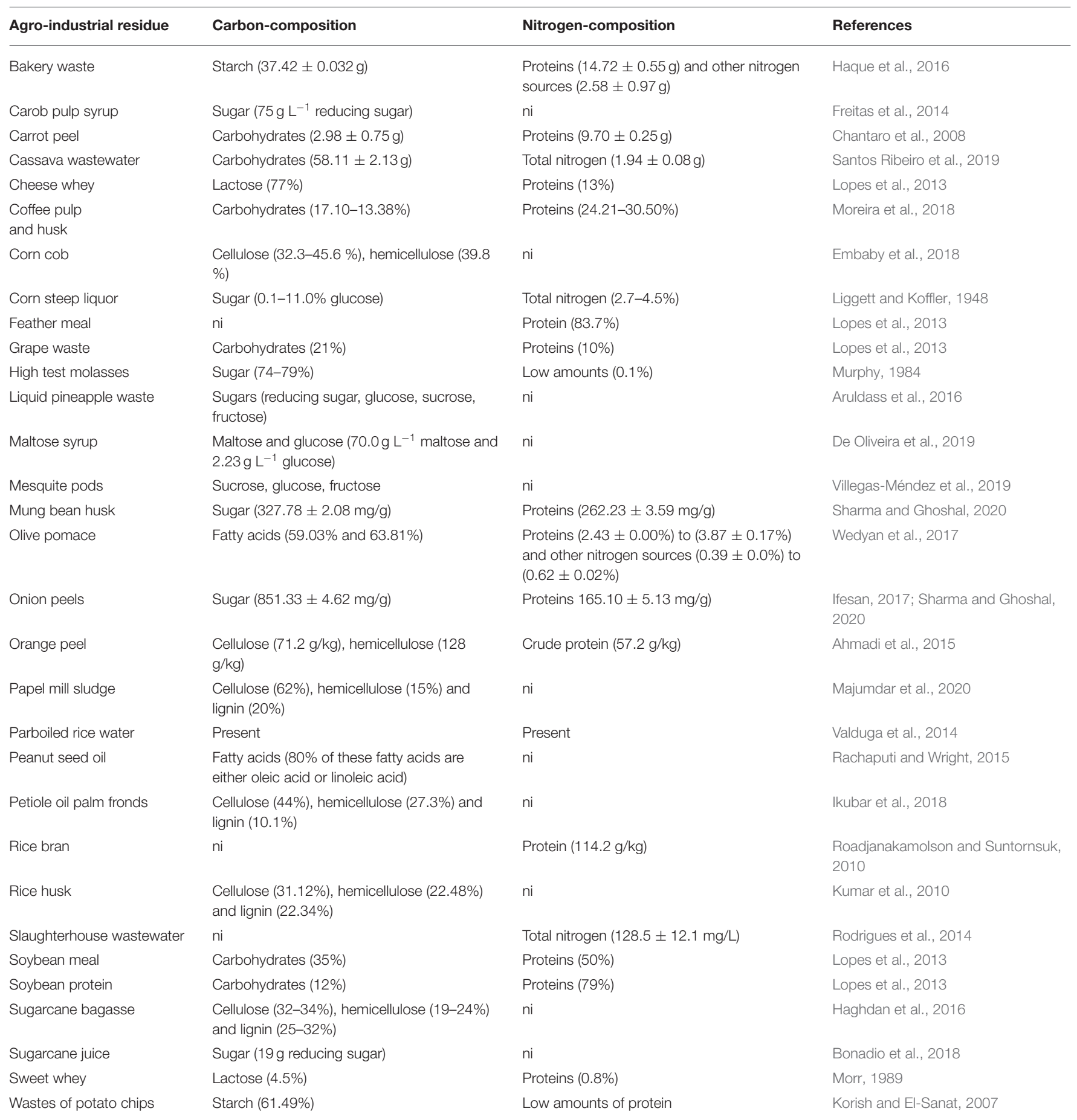

ni, not informed.

fermentation is suitable to filamentous fungi and microorganisms that require low moisture content for their growth. Their growth as SSF mimic (Joshi and Attri, 2006). This technique is very effective in waste valorization due to the microorganism conversion of waste into value-added products with low power supply and high yield. Arikan and co-authors observed higher production of a yellow pigment by Aspergillus carbonarius using pomegranate pulp through SSF than SmF (Arikan et al., 2020). According to Carvalho et al. (2005), the majority of Monascus researchers observed pigment production varying from hundreds of absorbance units per $\mathrm{mL}$ culture medium, in SmF; in contrast thousands of absorbance units/g dry substrate was observed 
in SSF (Carvalho et al., 2005). In some cases, however, the higher production using SSF requires longer times of culture in comparison with SmF. The low energy to perform a SSF is due to the simplified bioreactors used in this type of fermentation, mainly when we compare some types of SmF bioreactors, such as stirred tank, that requires high energy consumption to maintain constant agitation during the process. In addition, it is considered an easy process, with minimal pretreatment of the waste being necessary (sometimes not needed at all) and generating less wastewater (Wang and Yang, 2007; Arun et al., 2020). Some of the common substrates used in SSF are wheat bran, rice and rice straw, fruit and vegetable waste, paper pulp, bagasse, and coconut coir (Panesar et al., 2015).

On the other hand, in SmF the substrates are consumed very rapidly, then it is possible to replace or supplement the culture media (fed batch or continuous culture strategies are necessary in some cases), or to use fast-growing microorganisms during the process (Subramaniyam and Vimala, 2012). It is important to highlight that in some cases supplementation of the medium in SSF is also needed, because of the poor nature of the substrate or low availability of the nutrients in the beginning of the bioprocess (substrates that are not pretreated, for example). This type of fermentation is commonly used for bacteria, due to their necessity of higher moisture in the fermentation process (Subramaniyam and Vimala, 2012). Some common substrates used in $\mathrm{SmF}$ are soluble sugars, molasses, fruit and vegetable juices, and sewage/wastewater (Panesar et al., 2015). Based on the data of this review (Table 2), the majority of the studies use SmF to produce pigments instead of using SSF. Solid-state fermentation systems could present some advantages because of the potential with natural substrates. Nonetheless, pigment production is performed commercially almost entirely in SmF (Sánchez-Muñoz et al., 2020). The main obstacles to SSF are the low amenability of the process to regulation, the heterogeneous fermentation conditions and the low reproducibility of the results. Moreover, some difficulties to scale-up may also appear, as well as often unfeasible determination of biomass or complicated product purification by downstream processes (Hölker and Lenz, 2005).

Agro-industrial residues decrease the costs of the fermentation process. Maximization of the pigment production while decreasing the production costs has been the goal of current techniques applied to produce microbial pigments at large scale. Medium optimization is especially important to maximize the production. Optimizing the medium includes controlling the conditions of the fermentation, such as temperature, $\mathrm{pH}$, aeration, agitation, and media components. Process optimization techniques have used statistical experimental designs and response surface analysis with limited use of artificial intelligence (like genetic algorithms). Response surface methodology (RSM) is an effective approach for the process optimization in pigment production (Gharibzahedi et al., 2012; Sen et al., 2019).

Some studies reported in this review used statistical methodologies to optimize the production of pigments, with RSM being by far the most used. Sharma and Goshal optimized $\mathrm{pH}$, temperature, and agitation conditions in the production of carotenoids by $R$. mucilaginosa (Sharma and Ghoshal,
2020). Embaby and co-authors used a three-step optimization, including RSM, to study the concentration of glycerol and inoculum size in the production of orange and red pigments by M. purpureus using corn cobs (Embaby et al., 2018). Aruldass and co-authors used RSM to optimize the production of yellowishorange pigments by Chryseobacterium artocarpi. The authors used three independent variables: concentrations of liquid pineapple waste, L-tryptophan, and $\mathrm{KH}_{2} \mathrm{PO}_{4}$ (Aruldass et al., 2016). The production of astaxanthin by Xanthophyllomyces dendrorhous in olive pomace was optimized with RSM, testing temperature, moisture content and $\mathrm{pH}$ condition (Eryilmaz et al., 2016). Prodigiosin production by $S$. marcescens was optimized by RSM with three variables: glycerol bagasse, soybean peptone bagasse, and initial moisture content (Xia et al., 2016). Raw glycerol, steep liquor, and sugarcane molasses concentrations were tested in the optimization experiments of carotenoids production by Sporidiobolus pararoseus, as well as glycerol, corn steep liquor and parboiled rice water concentrations (Valduga et al., 2014; Machado and de Medeiros Burkert, 2015). Moisture, $\mathrm{pH}$, and carbon-to-nitrogen ratio were evaluated in the carotenoids production by $R$. glutinis using rice bran (Roadjanakamolson and Suntornsuk, 2010). The Taguchi methodology was applied to optimize the production of carotenoids by Xanthophyllomyces dendrorhous, using four variables: concentrations of mesquite pods extract, corn steep liquor, yeast extract, and malt extract (Villegas-Méndez et al., 2019).

Another approach used in some studies is the optimization of one factor at a time (OVAT). For instance, the authors evaluated $\mathrm{pH}$, temperature, and light on the carotenoids production by Planococcus sp. (Majumdar et al., 2020). Optimization of various factors such as $\mathrm{pH}$, growth temperature, incubation time and the addition of nitrogen components was evaluated on the production of $\beta$-carotene by Blakeslea trispora (Kaur et al., 2019). Five operational factors such as the initial moisture content of oil palm frond, initial $\mathrm{pH}$, supplementation of nitrogen source, the percentage of petiole to leaflet, and inoculum size were investigated on the red pigments production by $M$. purpureus (Said and Hamid, 2019). In the conventional approach OVAT, the conditions are optimized by changing one factor at a time while maintaining the other variables constant. This is a simple approach to implement and helps to choose the main variables. However, this methodology is time-consuming, because several experiments are necessary to study all the relevant conditions and also ignores the combined interaction(s) among the variables (Vishwanatha et al., 2010).

Variables that were most frequently evaluated in the optimization studies were $\mathrm{pH}$, substrate concentration, and initial moisture. The $\mathrm{pH}$ is particularly important in the pigments production. $\mathrm{pH}$ of the medium plays an important role in activating key enzymes involved in pigment production and excretion by $M$. pupureus CCT3802, according to Orozco and Kilikian (2008). In addition, $\mathrm{pH}$ influences the color of the pigments. According to De Oliveira and co-authors, $\mathrm{pH}$ values close to neutrality lead to the formation of red pigments by Monascus sp. (De Oliveira et al., 2019). On the other hand, medium $\mathrm{pH}$ around 3 results in the production of 
yellow pigments by Monascus anka (Shi et al., 2015). Substrate concentration is considered as an important variable to study, in particular the nitrogen source is a key regulation factor. It has been consistently shown that selective nitrogen sources largely influence the composition of Monascus pigments and this variable correlates with $\mathrm{pH}$ of the medium (Shi et al., 2015), highlighting the importance of the statistical methodologies to study the interactions among variables. Moisture is also a significant variable in SSF systems. Adequate water content of the substrate facilitates the oxygen transport, promoting microorganism growth. Nevertheless, excessive water content may lead to the reduction of oxygen transfer and diffusion because of substrate agglomeration, lowering the porosity of the substrate and increasing the risk of contamination, since an advantage of SSF is to select microorganisms that grow under low water content (Said and Hamid, 2019). Indeed, some filamentous fungi and yeasts are capable to grow at a water activity $\left(\mathrm{a}_{w}\right)$ of 0.61 , the lowest $a_{w}$ value for growth of microorganisms (Grant, 2004).

Large scale production with high yields and low costs of production are the biggest challenges faced by industry. Recent developments in molecular biology could be crucial. The genes responsible for the synthesis of several pigments have been cloned and recombinant DNA technology would be an alternative to overproduction of pigments. With the advances in the gene technology, the scientists intend to create cell factories for the production of pigments using the heterologous expression of biosynthetic pathways from already known or novel pigment producers (Malpartida and Hopwood, 1984; Pfeifer and Khosla, 2001; Venil et al., 2013). Martínez and co-authors reviewed the recombinant production of melanin by Escherichia coli, Pseudomonas putida, and Streptomyces kathirae (Martínez et al., 2019). In another work, the authors deleted the $15-\mathrm{kb}$ citrinin biosynthetic gene cluster in $M$. purpureus industrial strain KL-001, using CRISPR (Clustered Regularly Interspaced Short Palindromic Repeats)-Cas system, obtaining a mutant strain that did not produce the mycotoxin and also increased red pigment production in $2-5 \%$ (Liu et al., 2020). From a technical point of view, the vast majority of the studies reported in the present review, were at a laboratory scale. Nevertheless, certain pigments are in a more advanced stage of scaling up. Some studies used SmF bioreactors, such as stirred tank and bubble column (Bühler et al., 2013; Rodrigues et al., 2014; Aruldass et al., 2015, 2016; Colet et al., 2017; Sharma and Ghoshal, 2020). These more advanced studies are interesting, since they show the high potentialities of scaling up microbial pigments targeting a future industrial application.

Industrial scale synthesis of microbial pigments is mainly limited by the high costs of production, the co-production of toxins in some of these processes, and the resistance of the final product to extreme processes conditions, such as high temperature and extreme $\mathrm{pHs}$ (conditions that are found in industrial processes) (Narsing Rao et al., 2017). Some microbial pigments are produced industrially, such as $\beta$-carotene (Blakeslea trispora and Dunaliella salina) and lycopene (Blakeslea trispora), Monascus-derived pigments in Natural Red ${ }^{\mathrm{TM}}$ (Penicillium oxalicum), riboflavin (Aspergillus gossypii), phycocianin (Spirulina platensis), and astaxanthin (Paracoccus carotinifasciens and Haematococcus pluvialis) (Dufosse et al., 2014). The estimation of the universal food colorant market is anticipated to achieve 3.75 billion USD by 2022 (Venil et al., 2020b). Besides, the carotenoids (astaxanthin, betacarotene, canthaxanthin, lutein, lycopene, zeaxanthin) market in 2019/2020 is supposed to reach $\$ 1.5-1.8$ billion with annual growth rate of $3.9 \%$ (Barredo et al., 2017; Venil et al., 2020a). The global demand for natural pigments, including microbial pigments is high and tends to grow exponentially in the next years.

\section{OTHER BIOLOGICAL ACTIVITIES OF MICROBIAL PIGMENTS PRODUCED WITH AGRO-INDUSTRIAL WASTE}

Microbial pigments not only add color, but they also have interesting biological properties such as antioxidant, antimicrobial, anticancer, immunoregulation, antiinflammatory, antiproliferative, and immunosuppressive activities (among others) (Kirti et al., 2014; Manimala and Murugesan, 2014; Kumar et al., 2015; Sen et al., 2019; Muthusamy et al., 2020). Because of these pharmacological properties, there are many more advantages of using natural pigments over synthetic colorants (Venil et al., 2013).

The most common biological activities were antibacterial and antioxidant in the reviewed studies (Table 2). The antimicrobial activity of pigments can be a strategy to improve the source microbe ability of competing with other microorganisms (De Carvalho et al., 2014). The violet crude extract produced by Chromobacterium violaceum using liquid pineapple waste contains the pigments violacein and deoxyviolacein. This extract has antibacterial activity against Staphylococcus aureus ATCC 29213 and methicillin-resistant S. aureus (MRSA) (Aruldass et al., 2015). The extracted $\beta$-carotene produced by Planococcus sp. TRC1 using paper mill sludge and sugarcane bagasse was active against Bacillus subtilis, Salmonella enterica, Escherichia coli, and Proteus vulgaris. In addition, this pigment had antioxidant activity (Majumdar et al., 2020). Red pigment produced by Talaromyces purpureogenus using Bengal gram husk was active against B. cereus, B. subtilis, S. aureus, Micrococcus luteus, E. coli, Klebsiella pneumoniae, Listeria monocytogenes, and Salmonella typhimurium. The extracted pigment presented antioxidant activity and was non-toxic to the crustacean Artemia franciscana (Pandit et al., 2019). Carotenoids produced by $R$. mucilaginosa using solid coffee waste exhibited antioxidant and antimicrobial activities against pathogenic bacteria: Salmonella cholerasus, E. coli, S. aureus, and L. monocytogenes, as well as against toxigenic fungi like Aspergillus flavus, A. parasiticus, A. carbonarius, and A. ochraceus (Moreira et al., 2018). $\beta$-carotene produced by Blakeslea trispora using fruit and vegetable waste, astaxanthin produced by Xanthophyllomyces dendrorhous in olive pomace, and melanin produced by Pseudomonas sp. using cabbage waste, also presented antioxidant activity (Tarangini and Mishra, 2013; Eryilmaz et al., 2016; Kaur et al., 2019). 


\section{CONCLUSIONS AND CHALLENGES}

The use of agro-industrial residues as substrate for microbial pigment synthesis is a green, sustainable way of solving a pollution problem while cutting costs in the production of added value assets. Besides reducing the carbon footprint, microbial pigments also satisfy a growing demand for natural colorants. The majority of these pigments are also vegan, circumventing the need for animal-based colors (dairy and poultry-waste based microbe production would not be vegan, however).

Considering the advantages of using microorganisms as pigment factories, there is a growing need for biodiversity sampling, in search for new molecular entities (including greater color variety). Not only that, but genetic engineering and synthetic biology approaches are expected to provide strains with increased productivity and tolerance to cultivation conditions. Brazil is an interesting place to bioprospect new microorganisms, due to its great natural biodiversity and its agriculture-based economy, providing a large variety of agro-industrial waste to be studied.

Besides the use of a strain that produces high yields of pigments in an inexpensive medium, the current and future challenges in this area are related to the safety of the final products, due to the mycotoxin co-produced by some of the microorganisms. Technologies to produce microorganisms with these characteristic are available and in some cases, they are not expensive, such as the new genome- editing methodology

\section{REFERENCES}

Abdel-Raheam, H. E. F., Farag, M. K. A., Ragab, W. S. M., Ramadan, E.-S. A., and Mahmoud, N. M. (2016). Potato manufacturing wastes-a novel substrate for the production of natural pigments from Monascus purpureus. Assiut J. Agric. Sci. 47, 13-23. doi: 10.21608/ajas.2016.505

Ahmad, W. A., Yusof, N. Z., Nordin, N., Zakaria, Z. A., and Rezali, M. F. (2012). Production and characterization of violacein by locally isolated Chromobacterium violaceum grown in agricultural wastes. Appl. Biochem. Biotechnol. 167, 1220-1234. doi: 10.1007/s12010-012-9553-7

Ahmadi, F., Zamiri, M. J., Khorvash, M., Banihashemi, Z., and Bayat, A. R. (2015). Chemical composition and protein enrichment of orange peels and sugar beet pulp after fermentation by two Trichoderma species. Iran. J. Vet. Res. 16:25.

Arikan, E. B., Canli, O., Caro, Y., Dufossé, L., and Dizge, N. (2020). Production of bio-based pigments from food processing industry by-products (apple, pomegranate, black carrot, red beet pulps) using Aspergillus carbonarius. J. Fungi 6:240. doi: 10.3390/jof6040240

Aruldass, C. A., Aziz, A., Venil, C. K., Khasim, A. R., and Ahmad, W. A. (2016). Utilization of agro-industrial waste for the production of yellowishorange pigment from Chryseobacterium artocarpi CECT 8497. Int. Biodeterior. Biodegradation 113, 342-349. doi: 10.1016/j.ibiod.2016.01.024

Aruldass, C. A., Venil, C. K., Ahmad, W. A., and others (2015). Violet pigment production from liquid pineapple waste by Chromobacterium violaceum UTM5 and evaluation of its bioactivity. RSC Adv. 5, 51524-51536. doi: 10.1039/C5RA05765E

Arun, K. B., Madhavan, A., Sindhu, R., Binod, P., Pandey, A., Reshmy, R., et al. (2020). Remodeling agro-industrial and food wastes into value-added bioactives and biopolymers. Ind. Crops Prod. 154:112621. doi: 10.1016/j.indcrop.2020.112621

Babitha, S. (2009). "Microbial pigments," in Biotechnology for Agro-Industrial Residues Utilisation, eds P. S. nee' Nigam and A. Pandey (Dordrecht: Springer), 147-162. doi: 10.1007/978-1-4020-9942-7_8
CRISPR. Another important approach is the stability of the natural pigments that are in some cases a problem for the final product. More studies about different modes of production are needed in order to find new pigments with high stability to be applied in different industries. Lastly, the use of agro-industrial residues needs to be more implemented in industries not only to decrease the costs of the production but also to execute a circular economy, that is an eco-friendly approach, extremely necessary in the current days.

The road is still long and largely unpaved for microbe-based biopigments, and as their use expands, so will the development of associated technologies with proportional cost reduction. In summary, the microbial pigment production using agroindustrial residues is a rare win-win scenario, in which "One man's trash is another man's treasure."

\section{AUTHOR CONTRIBUTIONS}

All authors listed have made a substantial, direct and intellectual contribution to the work, and approved it for publication.

\section{ACKNOWLEDGMENTS}

We would like to thank the Brazilian agency Conselho de Aperfeiçoamento de Pessoal de Nível Superior (CAPES) by the postdoctoral fellowship (FCL).

Barredo, J. L., García-Estrada, C., Kosalkova, K., and Barreiro, C. (2017). Biosynthesis of astaxanthin as a main carotenoid in the heterobasidiomycetous yeast Xanthophyllomyces dendrorhous. J. Fungi 3:44. doi: 10.3390/jof3030044

Bhosale, P., and Gadre, R. V. (2001). Optimization of carotenoid production from hyper-producing Rhodotorula glutinis mutant 32 by a factorial approach. Lett. Appl. Microbiol. 33, 12-16. doi: 10.1046/j.1472-765X.2001. 00940.x

Blanc, P., Laussac, J. P., Le Bars, J., Le Bars, P., Loret, M. O., Pareilleux, A., et al. (1995). Characterization of monascidin A from Monascus as citrinin. Int. J. Food Microbiol. 27, 201-213. doi: 10.1016/0168-1605(94)00167-5

Bonadio, M. de P., Freitas, L. A. de, and Mutton, M. J. R. (2018). Carotenoid production in sugarcane juice and synthetic media supplemented with nutrients by Rhodotorula rubra 102. Brazilian J. Microbiol. 49, 872-878. doi: 10.1016/j.bjm.2018.02.010

Bühler, R. M. M., Dutra, A. C., Vendruscolo, F., Moritz, D. E., and Ninow, J. L. (2013). Monascus pigment production in bioreactor using a co-product of biodiesel as substrate. Food Sci. Technol. 33, 9-13. doi: 10.1590/S0101-20612013000500002

Carvalho, J. C., de Oishi, B. O., Pandey, A., and Soccol, C. R. (2005). Biopigments from Monascus: strains selection, citrinin production and color stability. Brazilian Arch. Biol. Technol. 48, 885-894. doi: 10.1590/S1516-89132005000800004

Chantaro, P., Devahastin, S., and Chiewchan, N. (2008). Production of antioxidant high dietary fiber powder from carrot peels. LWT-Food Sci. Technol. 41, 1987-1994. doi: 10.1016/j.lwt.2007.11.013

Charalampia, D., Antonios, K. E., Constantina, N., and Haralabos, K. C. (2017). Current trends and emerging technologies in biopigment production processes: industrial food and health applications. Int. J. Hortic. Agric. Food Sci. 1, 33-46. Available online at: https://www.academia.edu/35858011/Current_ trends_and_emerging_technologies_in_biopigment_production_processes_ Industrial_food_and_health_applications

Colet, R., Urnau, L., Bampi, J., Zeni, J., Dias, B. B., Rodrigues, E., et al. (2017). Use of low-cost agro products as substrate in semi-continuous process to 
obtain carotenoids by Sporidiobolus salmonicolor. Biocatal. Agric. Biotechnol. 11, 268-274. doi: 10.1016/j.bcab.2017.07.015

Da Silva Vilar, D., Cruz, I. A., Torres, N. H., Figueiredo, R. T., de Melo, L., de Resende, I. T. F., et al. (2019). "Agro-industrial wastes: environmental toxicology, risks, and biological treatment approaches," in Environmental Contaminants: Ecological Implications and Management, ed R. Naresh Bharagava (Singapore: Springer), 1-23. doi: 10.1007/978-981-13-7904-8_1

Daud, N. F. S., Said, F. M., Ramu, M., and Yasin, N. M. H. (2020). Evaluation of bio-red pigment extraction from Monascus purpureus FTC5357. MSandE 736:22084. doi: 10.1088/1757-899X/736/2/022084

De Carvalho, J. C., Cardoso, L. C., Ghiggi, V., Woiciechowski, A. L., de Souza Vandenberghe, L. P., and Soccol, C. R. (2014). "Microbial pigments," in Biotransformation of Waste Biomass into High Value Biochemicals, eds S. K. Brar, G. S. Dhillon, and M. Fernandes (New York, NY: Springer), 73-97. doi: 10.1007/978-1-4614-8005-1_4

De Oliveira, C. F. D., da Costa, J. P. V., and Vendruscolo, F. (2019). Maltose syrup residue as the substrate for Monascus pigments production. Biocatal. Agric. Biotechnol. 18:101101. doi: 10.1016/j.bcab.2019.101101

Dufossé, L. (2006). Microbial production of food grade pigments. Food Technol. Biotechnol. 44, 313-321. doi: 10.1016/b978-0-12-809633-8.13091-2

Dufosse, L., Fouillaud, M., Caro, Y., Mapari, S. A. S., and Sutthiwong, N. (2014). Filamentous fungi are large-scale producers of pigments and colorants for the food industry. Curr. Opin. Biotechnol. 26, 56-61. doi: 10.1016/j.copbio.2013.09.007

Embaby, A. M., Hussein, M. N., and Hussein, A. (2018). Monascus orange and red pigments production by Monascus purpureus ATCC16436 through co-solid state fermentation of corn cob and glycerol: an eco-friendly environmental low cost approach. PLoS ONE 13:e0207755. doi: 10.1371/journal.pone. 0207755

Eryilmaz, E. B., Dursun, D., and Dalgiç, A. C. (2016). Multiple optimization and statistical evaluation of astaxanthin production utilizing olive pomace. Biocatal. Agric. Biotechnol. 7, 224-227. doi: 10.1016/j.bcab.2016. 06.012

Freitas, C., Parreira, T. M., Roseiro, J., Reis, A., and da Silva, T. L. (2014). Selecting low-cost carbon sources for carotenoid and lipid production by the pink yeast Rhodosporidium toruloides NCYC 921 using flow cytometry. Bioresour. Technol. 158, 355-359. doi: 10.1016/j.biortech.2014.02.071

Galal, G. F., and Ahmed, R. F. (2020). Using of some agro-industrial wastes for improving carotenoids production from yeast Rhodotorula glutinis 32 and bacteria Erwinia uredovora DSMZ 30080. Microbiol. Res. J. Int. 30, 15-25. doi: $10.9734 / \mathrm{mrji} / 2020 / \mathrm{v} 30 \mathrm{i} 130186$

Gharibzahedi, S. M. T., Mousavi, S. M., Hamedi, M., Khodaiyan, F., and Razavi, S. H. (2012). Development of an optimal formulation for oxidative stability of walnut-beverage emulsions based on gum arabic and xanthan gum using response surface methodology. Carbohydr. Polym. 87, 1611-1619. doi: 10.1016/j.carbpol.2011.09.067

Goswami, G., Chaudhuri, S., and Dutta, D. (2015). Studies on the stability of a carotenoid produced by a novel isolate using low cost agro-industrial residue and its application in different model systems. LWT-Food Sci. Technol. 63, 780-790. doi: 10.1016/j.lwt.2015.03.017

Grant, W. D. (2004). Life at low water activity. Philos. Trans. R. Soc. London 359, 1249-1267. doi: 10.1098/rstb.2004.1502

Haghdan, S., Renneckar, S., Smith, G.D. (2016). "Sources of lignin," in Lignin in Polymer Composites, eds O. Faruk and M. Sain (Vancouver, BC: Elsevier), 1-11.

Haque, M. A., Kachrimanidou, V., Koutinas, A., and Lin, C. S. K. (2016). Valorization of bakery waste for biocolorant and enzyme production by Monascus purpureus. J. Biotechnol. 231, 55-64. doi: $10.1016 /$ j.jbiotec.2016.05.003

Heredia-Guerrero, J. A., Heredia, A., Dominguez, E., Cingolani, R., Bayer, I. S., Athanassiou, A., et al. (2017). Cutin from agro-waste as a raw material for the production of bioplastics. J. Exp. Bot. 68, 5401-5410. doi: 10.1093/jxb/ erx 272

Hernández-Velasco, P., Morales-Atilano, I., Rodríguez-Delgado, M., RodríguezDelgado, J. M., Luna-Moreno, D., Ávalos-Alanís, F. G., et al. (2020). Photoelectric evaluation of dye-sensitized solar cells based on prodigiosin pigment derived from Serratia marcescens 11E. Dye. Pigment. 177:108278. doi: 10.1016/j.dyepig.2020.108278
Hilares, R. T., de Souza, R. A., Marcelino, P. F., da Silva, S. S., Dragone, G., Mussatto, S. I., et al. (2018). Sugarcane bagasse hydrolysate as a potential feedstock for red pigment production by Monascus ruber. Food Chem. 245, 786-791. doi: 10.1016/j.foodchem.2017.11.111

Hölker, U., and Lenz, J. (2005). Solid-state fermentation - are there any biotechnological advantages? Curr. Opin. Microbiol. 8, 301-306. doi: $10.1016 /$ j.mib.2005.04.006

Ifesan, B. O. T. (2017). Chemical composition of onion peel (Allium cepa) and its ability to serve as a preservative in cooked beef. Int. J. Sci. Res. Methodol. 7, 1-10.

Ikubar, M. R. M., Manan, M. A., Salleh, M. M., and Yahya, A. (2018). Solidstate fermentation of oil palm frond petiole for lignin peroxidase and xylanase-rich cocktail production. 3 Biotech 8:259. doi: 10.1007/s13205-0181268-1

Joshi, V. K., and Attri, D. (2006). Solid state fermentation of apple pomace for the production of value added products. Nat. Prod. Rad. 5, 289-296.

Kantifedaki, A., Kachrimanidou, V., Mallouchos, A., Papanikolaou, S., and Koutinas, A. A. (2018). Orange processing waste valorisation for the production of bio-based pigments using the fungal strains Monascus purpureus and Penicillium purpurogenum. J. Clean. Prod. 185, 882-890. doi: 10.1016/j.jclepro.2018.03.032

Kaur, P., Ghoshal, G., and Jain, A. (2019). Bio-utilization of fruits and vegetables waste to produce $\beta$-carotene in solid-state fermentation: characterization and antioxidant activity. Process Biochem. 76, 155-164. doi: 10.1016/j.procbio.2018.10.007

Kirti, K., Amita, S., Priti, S., Mukesh Kumar, A., and Jyoti, S. (2014). Colorful world of microbes: carotenoids and their applications. Adv. Biol. 2014:837891. doi: $10.1155 / 2014 / 837891$

Korish, M., and El-Sanat, S. (2007). Utilization of Potato-Chips Waste for Production of High Economic Value Products: I-Production of Baker's Yeast. Alexandria.

Kumar, A., Vishwakarma, H. S., Singh, J., Dwivedi, S., and Kumar, M. (2015). Microbial pigments: production and their applications in various industries. Int. J. Pharm. Chem. Biol. Sci. 5, 203-212.

Kumar, P. S., Ramakrishnan, K., Kirupha, S. D., and Sivanesan, S. (2010). Thermodynamic and kinetic studies of cadmium adsorption from aqueous solution onto rice husk. Brazilian J. Chem. Eng. 27, 347-355. doi: 10.1590/S0104-66322010000200013

Lagashetti, A. C., Dufossé, L., Singh, S. K., and Singh, P. N. (2019). Fungal pigments and their prospects in different industries. Microorganisms 7:604. doi: 10.3390/microorganisms7120604

Liggett, R. W., and Koffler, H. (1948). Corn steep liquor in microbiology. Bacteriol. Rev. 12:297. doi: 10.1128/BR.12.4.297-311.1948

Liu, W., An, C., Shu, X., Meng, X., Yao, Y., Zhang, J., et al. (2020). A dual-plasmid CRISPR/Cas system for mycotoxin elimination in polykaryotic industrial fungi. ACS Synth. Biol. 9, 2087-2095. doi: 10.1021/acssynbio. 0c00178

Lopes, F. C., Tichota, D. M., Pereira, J. Q., Segalin, J., De Oliveira Rios, A., and Brandelli, A. (2013). Pigment production by filamentous fungi on agroindustrial byproducts: an eco-friendly alternative. Appl. Biochem. Biotechnol. 171, 616-625. doi: 10.1007/s12010-013-0392-y

Machado, W. R. C., and de Medeiros Burkert, J. F. (2015). Optimization of agroindustrial medium for the production of carotenoids by wild yeast Sporidiobolus pararoseus. African J. Microbiol. Res. 9, 209-219. doi: 10.5897/AJMR2014.7096

Madeira Jr., J. V., Contesini, F. J., Calzado, F., Rubio, M. V., Zubieta, M. P., Lopes, D. B., et al. (2017). "Agro-industrial residues and microbial enzymes: an overview on the eco-friendly bioconversion into high value-added products," in Biotechnology of Microbial Enzymes, ed G. Brahmachari (London: Elsevier), 475-511. doi: 10.1016/B978-0-12-803725-6.00018-2

Majumdar, S., Mandal, T., and Dasgupta Mandal, D. (2020). Production kinetics of $\beta$-carotene from Planococcus sp. TRC1 with concomitant bioconversion of industrial solid waste into crystalline cellulose rich biomass. Process Biochem. 92, 202-213. doi: 10.1016/j.procbio.2020.01.012

Malik, K., Tokkas, J., and Goyal, S. (2012). Microbial pigments: a review. Int. J. Microb. Res. Technol. 1, 361-365.

Malpartida, F., and Hopwood, D. A. (1984). Molecular cloning of the whole biosynthetic pathway of a Streptomyces antibiotic and its expression in a heterologous host. Nature 309, 462-464. doi: 10.1038/309462a0 
Manimala, M. R. A., and Murugesan, R. (2014). In vitro antioxidant and antimicrobial activity of carotenoid pigment extracted from Sporobolomyces sp. isolated from natural source. J. Appl. Nat. Sci. 6, 649-653. doi: 10.31018/jans.v6i2.511

Manimala, M. R. A., and Murugesan, R. (2017). Studies on carotenoid pigment production by yeast Rhodotorula mucilaginosa using cheap materials of agroindustrial origin. Pharma Innov. 6:80.

Martínez, L. M., Martinez, A., and Gosset, G. (2019). Production of melanins with recombinant microorganisms. Front. Bioeng. Biotechnol. 7:285. doi: 10.3389/fbioe.2019.00285

Moreira, M. D., Melo, M. M., Coimbra, J. M., dos Reis, K. C., Schwan, R. F., and Silva, C. F. (2018). Solid coffee waste as alternative to produce carotenoids with antioxidant and antimicrobial activities. Waste Manag. 82, 93-99. doi: 10.1016/j.wasman.2018.10.017

Morr, C. V. (1989). Whey proteins: manufacture. Dev. Dairy Chem. 4, 245-284.

Murphy, N. F. (1984). Fermentation of high test molasses. J. Agric. Univ. Puerto Rico 68, 33-44. doi: 10.46429/jaupr.v68i1.7256

Muthusamy, S., Udhayabaskar, S., Udayakumar, G. P., Kirthikaa, G. B., and Sivarajasekar, N. (2020). "Properties and applications of natural pigments produced from different biological sources-a concise review," in Sustainable Development in Energy and Environment, eds V. Sivasubramanian, A. Pugazhendhi, and I. Moorthy (Singapore: Springer). doi: 10.1007/978-981-15-4638-9 9

Narsing Rao, M. P., Xiao, M., and Li, W.-J. (2017). Fungal and bacterial pigments: secondary metabolites with wide applications. Front. Microbiol. 8:1113. doi: $10.3389 /$ fmicb.2017.01113

Nayak, A., and Bhushan, B. (2019). An overview of the recent trends on the waste valorization techniques for food wastes. J. Environ. Manage. 233, 352-370. doi: 10.1016/j.jenvman.2018.12.041

nee'Nigam, P. S., Gupta, N., and Anthwal, A. (2009). "Pre-treatment of agroindustrial residues," in Biotechnology for Agro-Industrial Residues Utilisation, eds P. S. nee' Nigam and A. Pandey (Dordrecht: Springer), 13-33. doi: 10.1007/978-1-4020-9942-7_2

Nigam, P. S., and Luke, J. S. (2016). Food additives: production of microbial pigments and their antioxidant properties. Curr. Opin. Food Sci. 7, 93-100. doi: 10.1016/j.cofs.2016.02.004

Nimnoi, P., and Lumyong, S. (2011). Improving solid-state fermentation of Monascus purpureus on agricultural products for pigment production. Food Bioprocess Technol. 4, 1384-1390. doi: 10.1007/s11947-0090233-8

Orozco, S. F. B., and Kilikian, B. V. (2008). Effect of pH on citrinin and red pigments production by Monascus purpureus CCT3802. World J. Microbiol. Biotechnol. 24, 263-268. doi: 10.1007/s11274-007-9465-9

Pandit, S. G., Ramesh, K. P. M., Puttananjaiah, M. H., and Dhale, M. A. (2019). Cicer arietinum (Bengal gram) husk as alternative for Talaromyces purpureogenus CFRM02 pigment production: bioactivities and identification. LWT 116:108499. doi: 10.1016/j.lwt.2019.108499

Panesar, R., Kaur, S., and Panesar, P. S. (2015). Production of microbial pigments utilizing agro-industrial waste: a review. Curr. Opin. Food Sci. 1, 70-76. doi: 10.1016/j.cofs.2014.12.002

Pfeifer, B. A., and Khosla, C. (2001). Biosynthesis of polyketides in heterologous hosts. Microbiol. Mol. Biol. Rev. 65, 106-118. doi: 10.1128/MMBR.65.1.106-118.2001

Rachaputi, R. C. N., and Wright, G. (2015). "Peanuts: overview," in Encyclopedia of Food Grains, eds C. Wrigley, H. Corke, K. Seetharaman, and J. Faubion (Oxford: Elsevier), 334-340. doi: 10.1016/B978-0-08-100596-5.00038-X

Roadjanakamolson, M., and Suntornsuk, W. (2010). Production of $\beta$ carotene-enriched rice bran using solid-state fermentation of Rhodotorula glutinis. J. Microbiol. Biotechnol. 20, 525-531. doi: 10.4014/jmb.0809. 0550

Rodrigues, D. B., Flores, É. M. M., Barin, J. S., Mercadante, A. Z., JacobLopes, E., and Zepka, L. Q. (2014). Production of carotenoids from microalgae cultivated using agroindustrial wastes. Food Res. Int. 65, 144-148. doi: 10.1016/j.foodres.2014.06.037

Sadh, P. K., Duhan, S., and Duhan, J. S. (2018). Agro-industrial wastes and their utilization using solid state fermentation: a review. Bioresour. Bioprocess. 5:1. doi: 10.1186/s40643-017-0187-z
Said, F. M., and Hamid, N. O. R. F. (2019). Natural red colorant via solid-state fermentation of oil palm frond by Monascus purpureus FTC 5356: effect of operating factors. J. Eng. Sci. Technol. 14, 2576-2589.

Sánchez, C. (2009). Lignocellulosic residues: biodegradation and bioconversion by fungi. Biotechnol. Adv. 27, 185-194. doi: 10.1016/j.biotechadv.2008. 11.001

Sánchez-Muñoz, S., Mariano-Silva, G., Leite, M. O., Mura, F. B., Verma, M. L., da Silva, S. S., et al. (2020). "Production of fungal and bacterial pigments and their applications," in Biotechnological Production of Bioactive Compounds, eds M. L. Verma and A. K. Chandel (Amsterdam: Elsevier), 327-361. doi: 10.1016/B978-0-444-64323-0.00011-4

Santos Ribeiro, J. E., da Silva Sant'Ana, A. M., Martini, M., Sorce, C., Andreucci, A., de Melo, D. J., et al. (2019). Rhodotorula glutinis cultivation on cassava wastewater for carotenoids and fatty acids generation. Biocat. Agric. Biotechnol. 22, 1-8. doi: 10.1016/j.bcab.2019.101419

Schneider, T., Graeff-Hönninger, S., French, W. T., Hernandez, R., Merkt, N., Claupein, W., et al. (2013). Lipid and carotenoid production by oleaginous red yeast Rhodotorula glutinis cultivated on brewery effluents. Energy 61, 34-43. doi: 10.1016/j.energy.2012.12.026

Sen, T., Barrow, C. J., and Deshmukh, S. K. (2019). Microbial pigments in the food industry-Challenges and the way forward. Front. Nutr. 6:7. doi: 10.3389/fnut.2019.00007

Sharma, R., and Ghoshal, G. (2020). Optimization of carotenoids production by Rhodotorula mucilaginosa (MTCC-1403) using agro-industrial waste in bioreactor: a statistical approach. Biotechnol. Reports 25:e00407. doi: 10.1016/j.btre.2019.e00407

Shi, K., Song, D., Chen, G., Pistolozzi, M., Wu, Z., and Quan, L. (2015). Controlling composition and color characteristics of Monascus pigments by $\mathrm{pH}$ and nitrogen sources in submerged fermentation. J. Biosci. Bioeng. 120, 145-154. doi: 10.1016/j.jbiosc.2015.01.001

Sopandi, T., Wardah, A., Surtiningsih, T., Suwandi, A., and Smith, J. J. (2013). Utilization and optimization of a waste stream cellulose culture medium for pigment production by Penicillium spp. J. Appl. Microbiol. 114, 733-745. doi: $10.1111 /$ jam. 12110

Subramaniyam, R., and Vimala, R. (2012). Solid state and submerged fermentation for the production of bioactive substances: a comparative study. Int. J. Sci. Nat. $3,480-486$

Tarangini, K., and Mishra, S. (2013). Production, characterization and analysis of melanin from isolated marine Pseudomonas sp. using vegetable waste. Res. J. Eng. Sci. 2, 40-46.

Taskin, M., Sisman, T., Erdal, S., and Kurbanoglu, E. B. (2011). Use of waste chicken feathers as peptone for production of carotenoids in submerged culture of Rhodotorula glutinis MT-5. Eur. Food Res. Technol. 233:657. doi: 10.1007/s00217-011-1561-2

Valduga, E., Ribeiro, A. H. R., Cence, K., Colet, R., Tiggemann, L., Zeni, J., et al. (2014). Carotenoids production from a newly isolated Sporidiobolus pararoseus strain using agroindustrial substrates. Biocatal. Agric. Biotechnol. 3, 207-213. doi: $10.1016 /$ j.bcab.2013.10.001

Vandamme, E. J. (2009). "Agro-industrial residue utilization for industrial biotechnology products," in Biotechnology for Agro-Industrial Residues Utilisation eds P. S. nee' Nigam and A. Pandey (Dordrecht: Springer), 3-11. doi: 10.1007/978-1-4020-9942-7_1

Velmurugan, P., Hur, H., Balachandar, V., Kamala-Kannan, S., Lee, K.-J., Lee, S.-M., et al. (2011). Monascus pigment production by solid-state fermentation with corn cob substrate. J. Biosci. Bioeng. 112, 590-594. doi: 10.1016/j.jbiosc.2011.08.009

Venil, C. K., Devi, P. R., and Ahmad, W. A. (2020b). "Agro-industrial waste as substrates for the production of bacterial pigment," in Valorisation of Agro-industrial Residues-Volume I: Biological Approaches, eds Z. Akmar Zakaria, R. Boopathy, J. R. Dib (Switzerland: Springer), 149-162. doi: 10.1007/978-3-030-39137-9_7

Venil, C. K., Dufossé, L., and Renuka Devi, P. (2020a). Bacterial Pigments: sustainable compounds with market potential for pharma and food industry. Front. Sustain. Food Syst. 4:100. doi: 10.3389/fsufs.2020.00100

Venil, C. K., Zakaria, Z. A., and Ahmad, W. A. (2013). Bacterial pigments and their applications. Process Biochem. 48, 1065-1079. doi: 10.1016/j.procbio.2013.06.006 
Villegas-Méndez, M. Á., Aguilar-Machado, D. E., Balagurusamy, N., Montañez, J., and Morales-Oyervides, L. (2019). Agro-industrial wastes for the synthesis of carotenoids by Xanthophyllomyces dendrorhous: mesquite podsbased medium design and optimization. Biochem. Eng. J. 150:107260. doi: 10.1016/j.bej.2019.107260

Vishwanatha, K. S., Rao, A. G. A., and Singh, S. A. (2010). Acid protease production by solid-state fermentation using Aspergillus oryzae MTCC 5341: optimization of process parameters. J. Ind. Microbiol. Biotechnol. 37, 129-138. doi: 10.1007/s10295-009-0654-4

Wang, L., and Yang, S.-T. (2007). "Solid state fermentation and its applications," in Bioprocessing for Value-Added Products from Renewable Resources, ed S.-T. Yang (Oxford: Elsevier), 465-489. doi: 10.1016/B978-044452114-9/ 50019-0

Wedyan, M., Hanieh, B. A., and Al Harahsheh, A. (2017). Chemical characterization of olive pomace in the northern region of Jordan. Bulgar. J. Agr. Sci. 23, 866-872. doi: 10.20944/preprints201703.0150.v1

Xia, Y., Wang, G., Lin, X., Song, X., and Ai, L. (2016). Solid-state fermentation with Serratia marcescens Xd-1 enhanced production of prodigiosin by using bagasse as an inertia matrix. Ann. Microbiol. 66, 1239-1247. doi: 10.1007/s13213-016-1208-4

Zihare, L., Spalvins, K., and Blumberga, D. (2018). Multi criteria analysis for products derived from agro-industrial by-products. Energy Procedia 147, 452-457. doi: 10.1016/j.egypro.2018. 07.045

Conflict of Interest: The authors declare that the research was conducted in the absence of any commercial or financial relationships that could be construed as a potential conflict of interest.

Copyright (c) 2021 Lopes and Ligabue-Braun. This is an open-access article distributed under the terms of the Creative Commons Attribution License (CC BY). The use, distribution or reproduction in other forums is permitted, provided the original author(s) and the copyright owner(s) are credited and that the original publication in this journal is cited, in accordance with accepted academic practice. No use, distribution or reproduction is permitted which does not comply with these terms. 